NASA Technical Memorandum 102525

ICOMP-90-07

\title{
Unsteady Three-Dimensional Marginal Separation, Including Breakdown
}

Peter W. Duck

University of Manchester

Manchester, England

and Institute for Computational Mechanics in Propulsion

Lewis Research Center

Cleveland, Ohio

(NACA-TM-10252S) UNSTEACY THREE-NIMENSIONAL

N90-20337

MAFEINAL SEPAPATITN, INCLUUTNG BREAKOOWN

(NASA) 310

CSCL 200

Unclas

$03 / 34$

0275317

February 1990

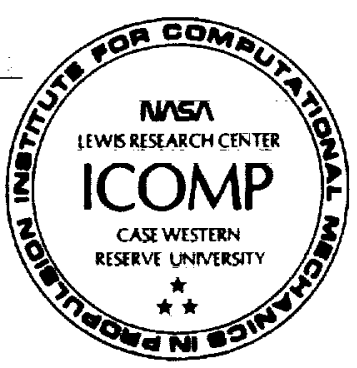


UNSTEADY THREE-DIMENSIONAL MARGINAL SEPARATION.

INCLUDING BREAKDOWN

Peter W. Duck

Department of Mathematics

University of Manchester

Manchester, England

and Institute for Computational Mechanics in Propulsion*

Lewis Research Center

Cleveland, Ohio

\section{Abstract}

We consider a situation involving a threc-dimensional marginal separation, where a (steady) boundary-layer flow is on the verge of separating at a point (located along a line of symetry/centreline). At this point we include a "triple-deck", thereby permitting a small amount of interaction to occur. Unsteadiness is included within this interaction region through some external means. It is shown that the problem reduces to the solution of a non-linear, unsteady, partialintegro system, which is solved numerically by means of time-marching logether with a pseudo-spectral method spatially. A number of solutions to this system are presented which strongly suggest a breakdown of this system may occur, at a finite spatial position, at a finite time. The structurc and details of this breakdown are then described.

*Work funded by Space Act Agreement C-99066-G. 


\section{Introduction}

In a recent paper, Duck (1989) (hereafter refered to as I), studied the effect of allowing a small amount of three-dimensional interaction (based on triple-deck theory) at a point where a steady laminar boundary layer was on the merge of separating. It was found that this interaction zone (i) permitted a small amount of reversed flow to occur within the interaction region and ( $i$ ) illustrated that non-uniqueness of solution was possible. These observations for the three-dimensional case were in line with related two-dimensional work published some years previously. The original work in this area was that of Stewartson, Smith and Kaups (1982), who analysed the (two-dimensional) problem; this was studied in further detail by Brown and Stewartson (1983), who showed that up to four solutions were possible for a particular choice of parameters.

This two-dimensional work was later extended to a three-dimensional situation, along a line of symmetry by Brown (1985), although, (as described in 1), a number of assumptions were made to simplify the computations, rendering the problem basically two-dimensional.

The work of Stewartson et al (1982) was extended to the unsteady regime by Smith (1982), who showed how the interacting flow may breakdown, with the development of a singularity at a point, at a finite time; this singularity was also described analytically (in addition to being observed numerically). Related studies of problems of this kind were then made by Ryzhov and Smith (1984), Smith and Elliott (1985), and extended to a rather more non-linear regime by Elliott and Smith (1987). These studies all confirmed the possibility of a finite-time breakdown of the solution. Further Goldstein, Leib and Cowley (1982) showed how two-dimensional Tollmien-Schlichting waves may be generated on marginally separated flow.

A number of other two-dimensional studies indicating the possible 
occurence of a finite-time singularity of a boundary layer in a tripledeck/interacting boundary layer include the studies of Smith and Bodonyi (1985), Duck (1985a), Duck (1985h), Duck (1987), Tutty and Cowley (1986). Recently Brotherton-Radcliffe and Smith (1987) and Smith (1988) have presented possible structures of such breakdowns. Further, Duck (1990) has presented a number of numerical results indicating the occurence of a finite-time breakdown in a three-dimensional non-linear, unsteady triple-deck flow.

In this paper we extend the three-dimensional work of I into the unsleady regime. As in $I$, we concern ourselves with a steady laminar boundary layer, which is on the verge of separating at a point, situated along a line of symmetry; in particular we assume that both the streamwise wall shear and the crossflow derivative of crossflow wall shear (directions defined relative to the freestream) vanish simultaneously at the point in question, but then both immediatly recover. This boundary layer could, for example, be set up on a body of revolution, as for cxample described by Cebeci, Khat tab and Stewartson (1980) and Cebeci and Su (1988). I'e then assume that this flow is perturbed in some (unsteady) fashion. Physically, this could perhaps be realised by some small amplitude pitching or yawing motion of the body (for example), although for this paper the particular details are not important.

We fully expect (guided by the two-dimensional results of Smith 1982) that the unsteady three-dimensional marginal separation problem may suffer finite-time breakdowns. The attractive feature of the problem is that this brcakdown is likcly to be partly analysable, and indeed, this turns out to be the casc; the author is unaware of any previous descriptions of a fully three-dimensional unsteady breakdown of an interacting boundary-layer problem.

The structure of the problem and the numerical techniques employed are 
broadly based on I, although the added dimension of time adds significantly to the computational complexity of the problem.

This particular problem is likely of practical importance, being linked to the problem of dyanamic stall on an aerofoil or on a body of revolution (for example), involving an abrupt change in the characteristics of the flow at some finitc angle of incidence (for example).

In the following section we go on to formulate the problem in detail. 


\section{Formulation}

Details here are similar in many respects to $I$, and consequently discussion of features similar in this study will be kept to a minimum.

We consider an incompressible fluid of kinematic viscosity $v$. We take $L$ to denote a typical lengthscale and $U_{\infty}$ a typical frecstram velocity directed along the line of symmetry of the configuration.

Cartesian coordinates $L(x, y, z)$ are chosen, with origin at the separation point; the velocity is then written as $U_{\infty}(u, v, w)$, and the pressure is $\rho_{\infty} U_{\infty}^{2} p, \rho_{\infty}$ being the fluid density. The line of symmetry corresponds to $z=0$, and the body surface (assuming insignificant curvature) is taken to lie along $y=0$.

The Reynolds number is defined as

$$
\mathrm{R}=\mathrm{U}_{\infty} \mathrm{L} / \mathrm{V},
$$

and this is taken to be large throughout this paper.

If a (typical) timescale of any unsteadiness is taken to be $T$, then we can define a second non-dimensional parameter, namely

$$
\mathrm{S}_{\mathrm{o}}=\mathrm{L} / \mathrm{TU}_{\infty} \text {, }
$$

and the non-dimensional equations governing the flow may be written as

$$
\nabla . \underline{u}=0 \text {, }
$$

So $\frac{\partial \underline{\mathrm{u}}}{\partial \mathrm{l}}+(\underline{\mathrm{u}} \cdot \nabla) \underset{\sim}{\mathrm{u}}=-\nabla \mathrm{p}+\mathrm{R}-1 \nabla^{2} \underset{\sim}{\mathrm{u}}$.

Symmetry of the flow about $z=0$ implies u,v,p are even functions about this plane, whilst $w$ must be an odd function of $z$. Throughout, on $y=0$, we impose the zero velocity condition $\underline{u}=\underline{\sim}$.

The details of the non-interaction zone are precisely the same as in I. If we define (standard) boundary-layer variables $Y$ and $V$ as follows

$$
y=R-\frac{1}{2} Y, \quad v=R-\frac{1}{1},
$$

then using the results found in $I$, as $x^{2}+z^{2} \rightarrow 0$, for $Y=0(1)$ 


$$
\begin{aligned}
u & =U_{0}(Y)+\mu r U_{0}{ }^{\prime}(Y)-x\left[w_{1}(Y)+V_{1}{ }^{\prime}(Y)\right] \\
& -1 x^{2}\left[V_{2}^{\prime}(Y)+w_{2}(Y)\right] \\
& +\mu^{2} r^{2} U_{0}{ }^{\prime}(Y)+z^{2} u_{23}(Y) \\
& -\mu x r\left[V_{1}{ }^{\prime}(Y)+w_{1}{ }^{\prime}(Y)\right]+\ldots \\
V & =v_{1}(Y)+\mu \frac{\lambda^{2} x}{r} U_{0}(Y) \\
& +x V_{2}(Y)-\mu^{2} \lambda^{2} x U_{0}(Y) \\
& -\mu r\left[w_{1}(Y)-V_{1}{ }^{\prime}(Y)\right] \\
& +x^{2} \frac{\lambda^{2} \mu}{r}\left[2 w_{1}(Y)+V_{1}^{\prime}(Y)\right]+\ldots, \\
w & =z w_{1}(Y)+z x w_{2}(Y)+\mu z r w_{1}(Y)+\ldots, \\
p & =P_{0}+P_{1} x+1 P_{2} x^{2}+\frac{1}{2} P_{3} z^{2}+\ldots
\end{aligned}
$$

Here we have written

$$
r=\left(\lambda^{2} x^{2}+z^{2}\right) \frac{1}{1}
$$

and we must have that

$$
\begin{aligned}
& U_{0}(0)=U_{0}^{\prime}(0)=v_{1}(0)=v_{1}^{\prime}(0) \\
& =w_{1}(0)=w_{1}(0)=v_{1}{ }^{\prime}(0) \\
& =w_{2}(0)=v_{2}(0)=0, \\
& v_{2}{ }^{\prime}(0)=\mu^{2} \lambda^{2} U_{0}{ }^{\prime}(0), \\
& u_{23}(0)=-\frac{1}{2} \mu^{2} U_{0}^{\prime}(0) .
\end{aligned}
$$

Further details are given in $I ; \mu$ and $\lambda$ may be regarded as parameters to the problem. The kcy outcome of the above equations is the cxistence of a discontinuity of derivatives of the solution as $x^{2}+z^{2} \rightarrow 0$. In order to alleviale this, we introduce a small interactive zone, where in

$$
\mathrm{X}=\mathrm{x} / \delta, \mathrm{Z}=\mathrm{z} / \delta \text {. }
$$

are the important streamwise and crossflow coordinates respectively, where

$$
\delta=R-1 / 5 \text {. }
$$

At this stage we must be specific about the magnitude of the unsteadiness parameler $S_{0}$. If unstcadiness is to play an important 
role in the problem (i.e. a time derivative is present in the key equation ( 2.31$)$, derived below), we must have that

$$
S_{0}=\delta^{1 / 4} S, S=0(1) \text {. }
$$

representing a relatively long timescale. A similar scaling was found by Smith (1982) to be important.

For $Y=0(1)$, the solution in the interaction zone is expected to develop as

$$
\begin{aligned}
u & =U_{0}(Y)+\delta\left\{-X\left[w_{1}(Y)+v_{1}^{\prime}(Y)\right]\right. \\
& \left.+A(X, Z, l) U_{0}^{\prime}(Y)\right\} \\
+ & \delta^{2}\left[-\frac{1}{2} X^{2}\left[V_{2}{ }^{\prime}(Y)+w_{2}(Y)\right]\right. \\
& +\left\lfloor\left[A(X, Z, t)^{2}+\gamma(Z, t)\right] U_{0}^{\prime}(Y)\right. \\
& \left.+Z^{2} u_{23}(Y)-X A(X, Z, t)\left[V_{1}^{\prime} \cdot(Y)+w_{1}(Y)\right]\right]+\ldots
\end{aligned}
$$

$$
\begin{aligned}
V= & V_{1}(Y)-A X U_{0}(Y) \\
+ & \delta\left\{X V_{2}(Y)-A A_{X} U_{0}^{\prime}(Y)\right. \\
& +(X A)_{X}\left[V_{1}^{\prime}(Y)+w_{1}(Y)\right] \\
& \left.-(Z A)_{Z} w_{1}(Y)\right)+\ldots \\
w= & \delta\left[Z w_{1}(Y)\right] \\
& +\delta^{2}\left\{X w_{2}(Y)+Z A(X, Z, 1) w_{1}^{\prime}(Y)\right\}+\ldots \\
P= & P_{0}+\delta P_{1} X+1 \delta^{2} P_{2} X^{2} \\
& +\frac{1}{2} \delta^{2} P_{3} Z^{2}+\delta^{5 / 2} \widetilde{P}(X, Z, t)+\ldots
\end{aligned}
$$

The central feature here is the inclusion of the arbitrary function $\gamma(Z, t)$, which replaces the arbitrary function of $Z$ (only) found in I. In order that a proper match is made with the non-interacting zone, we must have that

$$
\begin{aligned}
A(X, Z, t) & -\mu\left(\lambda^{2} X^{2}+Z^{2}\right) \\
& \text { as } X^{2}+Z^{2} \rightarrow \infty
\end{aligned}
$$

The solution (2.14) does not satisfy the no-slip condition on $Y=0$, and so it is necessary 10 include a further (viscous) layer 
("lower deck"). This is described by the layer wherein

$$
\widetilde{Y}=\mathrm{Y} \delta^{-1 / 4}=0(1)
$$

(see I, for example).

The solution then develops in a manner similar to (2.14) - (2.17), except (i) $Y$ is replaced by $\delta^{1 / 4} \tilde{Y}$, and (ii) there is a correction to the $u$ expansion of $\delta^{2} \tilde{U}(X, \bar{Y}, Z, t)$, to the $V$ expansion of $\delta^{5 / 4} \tilde{V}(X, \widetilde{Y}, Z, t)$, and to the $*$ expansion of $\delta^{2} \widetilde{W}(X, \widetilde{Y}, Z, t)$.

The governing equations for thesc correction terms are then

$$
\begin{aligned}
& \frac{\partial \widetilde{U}}{\partial \mathrm{X}}+\frac{\partial \widetilde{V}}{\partial \widetilde{Y}}+\frac{\partial \widetilde{W}}{\partial Z}=0 \text {, } \\
& \mathrm{S} \mathrm{a}_{2} \tilde{\mathrm{Y}} \frac{\partial \mathrm{A}}{\partial \mathrm{t}}+\frac{1}{\mathrm{a}_{2}} \mathrm{a}_{2} \tilde{\mathrm{Y}}^{2} \frac{\partial \tilde{U}}{\partial \mathrm{X}}+\mathrm{a}_{2} \tilde{\mathrm{Y}} \tilde{\mathrm{U}} \\
& =-\frac{\partial \tilde{P}}{\partial X}+\frac{\partial^{2} \tilde{U}}{\partial \tilde{Y}^{2}}, \\
& 1 a_{2} \tilde{Y}^{2} \frac{\partial \tilde{W}}{\partial X}=\frac{\partial^{2} \tilde{W}}{\partial \tilde{Y}^{2}}-\frac{\partial \widetilde{P}}{\partial Z},
\end{aligned}
$$

where $a_{2}=U_{0}^{\prime \prime}(0)$.

The boundary conditions to be applied to this system are

$$
\begin{aligned}
& \tilde{U}, \tilde{W} \rightarrow 0 \text { as } \tilde{Y} \rightarrow \infty, \\
& W(\tilde{Y}=0)=\tilde{V}(\tilde{Y}=0)=0,
\end{aligned}
$$

and

$$
\tilde{U}(\bar{Y}=0)=-\frac{a_{2}}{2}\left\{A^{2}+\gamma-\mu^{2}\left(\lambda^{2} X^{2}+Z^{2}\right)\right\}
$$

This system is similar to that found in I, but with the inclusion of the time derivative term in $(2.21)$. We now seck to determine the condition to be satisfied by $A(X, Z, t)$ if the above system ((2.20) (2.26)) has a solution.

Differentiating $(2.21)$ with respect to $X,(2.22)$ with respect to $Z$ and adding yields 


$$
\begin{aligned}
& 1 a_{2} Y^{2}\left[\frac{\partial^{2} \tilde{U}}{\partial X^{2}}+\frac{\partial^{2} \tilde{W}}{\partial X \partial Z}\right]+a_{2} \tilde{Y} \frac{\partial \tilde{Y}}{\partial X} \\
& =-\frac{\partial^{2} P}{\partial X^{2}}+\frac{\partial^{2} P}{\partial Z^{2}}+\frac{\partial^{3} \tilde{U}}{\partial \widetilde{Y} \partial X}+\frac{\partial^{2} \tilde{W}}{\partial \tilde{Y} \partial Z}-a_{2} \tilde{Y} S \frac{\partial^{2} A}{\partial X \partial t}
\end{aligned}
$$

Invoking continuity, namely (2.20), and differentiating (2.27) with respect to $\tilde{Y}$ yields

$$
\begin{gathered}
\frac{\partial^{4} \tilde{V}}{\partial \tilde{Y}^{4}}-a_{2} \tilde{Y}^{2} \frac{\partial^{3} \tilde{V}}{\partial \tilde{Y}^{2} \partial X}+a_{2} \frac{\partial \tilde{V}}{\partial X} \\
=-S a_{2} \frac{\partial^{2} A}{\partial X \partial t} .
\end{gathered}
$$

This equation must be solved subject to

$$
\tilde{\mathrm{V}} \rightarrow 0 \text { as } \tilde{\mathrm{Y}} \rightarrow \infty \text {, }
$$

whilst on $\tilde{\mathrm{Y}}=0$

$$
\begin{aligned}
& \tilde{V}=0, \\
& \frac{\partial \widetilde{V}}{\partial \widetilde{Y}}= \pm a_{2}\left[2 A A_{X}-2 \mu^{2} \lambda^{2} X\right], \\
& \frac{\partial^{3} \tilde{V}}{\partial \tilde{Y}^{3}}=-\left[\frac{\partial^{2} P}{\partial X^{2}}+\frac{\partial^{2} P}{\partial Z^{2}}\right] .
\end{aligned}
$$

Using the analyses of Stewartson (1970), Smith (1982) and I, we have that a solution to this system is only possible if the following condition is satisfied

$$
\begin{aligned}
& \frac{1}{2} a_{2}\left[A^{2}+\gamma(Z, t)-\mu^{2}\left(\lambda^{2} X^{2}+Z^{2}\right)\right]
\end{aligned}
$$

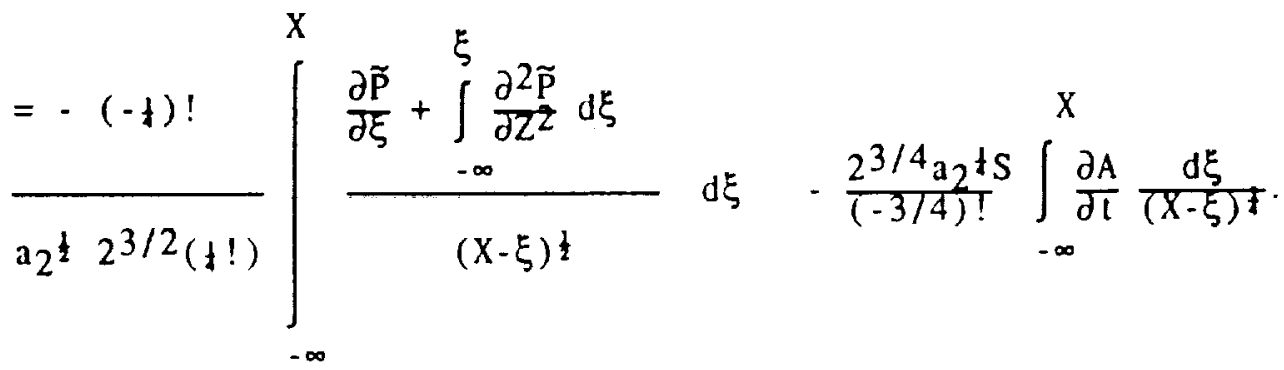


The system is closed by recourse to the upper deck, where $Y=0\left(\delta^{-3 / 2}\right.$ ) and this yields (Smith et al 1977, Duck and Burggraf 1986)

$$
\tilde{\mathrm{P}}(\mathrm{X}, \mathrm{Z}, 1)=-\frac{1}{2 \pi} \int_{-\infty}^{\infty} \int_{-\infty}^{\infty} \frac{\partial^{2} \mathrm{~A} / \partial \xi^{2} \mathrm{~d} \xi \mathrm{d} \zeta}{\left[(\mathrm{X}-\xi)^{2}+(\mathrm{Z}-\zeta)\right]^{2}}
$$

The combined system of (2.31) together with (2.32) represents a closed problem, (for prescribed $\gamma(Z, t)$ ). We discuss the numerical solution to this problem next. 


\section{Numerical Method}

Following 1, we choose to use a (double) Fourier Transform method to solve the system $(2.31)-(2.32)$. If we define the quanlity (for example)

$$
A^{* *}(k, l, t)=\int_{-\infty}^{\infty} \int_{-\infty}^{\infty} A(X, Z, t) e^{-i k X-i \ell Z} d X d Z
$$

then in $(k, l)$ space, $(2.31)=(2.32)$ may be easily combined to yield (after normalisation to remove positive constants)

$$
\begin{aligned}
& {\left[A^{2}+\gamma(Z, t)-\left(\lambda^{2} X^{2}+Z^{2}\right)\right]^{* *}} \\
& =-(i k) \frac{1}{2}\left(k^{2}+l^{2}\right) \frac{1}{A^{* *}}-(i k)^{-3 / 4} S A_{t}^{* *} .
\end{aligned}
$$

The numerical technique used in $I$ was based on assuming the right hand-side of (3.2) (without, of course the time derivative term) was known at each iteration level, and then an appropriate method was used to solve for the left-hand-side terms. Here, we anticipate using a lime marching scheme to handle time-variations; however such a scheme is likely to requite the time-derivative term to be included on the left-hand-side of (3.2), otherwise numerical instabilities are very likely to occur. Consequently, we choose to transfer the time-derivative term on to the left-hand-side of (3.2), and, in effect to express this term in physical $(X, Z)$ space, yielding

$$
\begin{aligned}
& {\left[A^{2}+\gamma(Z, t)-\left(\lambda^{2} X^{2}+Z^{2}\right)\right.} \\
& \left.+\frac{S}{\Gamma(3 / 4)} \int_{-\infty}^{X} \frac{A_{t}(\xi, Z, t) d \xi}{(X-\xi)^{t}}\right]^{* *} \\
& =-(i k)^{\frac{1}{2}}\left(k^{2}+l^{2}\right)^{\frac{1}{2}} A^{* *} .
\end{aligned}
$$

Further, just as in $I$, since $A(X, Z, t)$ is unbounded as $x^{2}+z^{2} \rightarrow \infty$, this function is inappropriate for numerical calculation (in particular for a Fouricr Transform method) in its present form. We follow $I$, and writc $A(X, Z, I)$ in the following form 
$A(X, Z, 1)=\rho-\frac{\gamma(Z, 1)+\alpha_{0}^{2}}{2 \rho}+A_{0}+B$

whe re $\rho=\left(\lambda^{2} x^{2}+Z^{2}+\alpha_{0}^{2}\right)^{\frac{1}{2}}$.

$$
\left(2 A_{o} \rho\right)^{* *}=-(i k)^{\frac{1}{2}}\left(k^{2}+l^{2}\right)\left\{\rho=\frac{\left[\gamma(Z, 1)+\alpha_{0}^{2}\right]}{2 \rho}\right\}^{* *}
$$

and $\alpha_{0}$ is a numerical constant which we have (artifically) introduced into the problem, to ensure boundedness as $x^{2}+z^{2} \rightarrow 0$ (indeed, in the limit of zero truncation error, the solution for $A(X, Z, t)$ is independent of $\alpha_{0}$.

We treat $B(X, Z, t)$ in (3.4) as our unknown, described by the equation

$$
\begin{aligned}
& \left\{\frac{\left[\gamma(Z, t)+\alpha_{0}^{2}\right]^{2}}{4 \rho^{2}}+2 B \rho\right. \\
& -\left[\gamma(Z, t)+\alpha_{0}^{2}\right]\left[\frac{A_{0}}{\rho}+\frac{B}{\rho}\right]+A_{0}^{2} \\
& \left.+2 A_{0} B+B^{2}+\frac{S}{\Gamma(3 / 4)} \int \frac{\left(A_{0}+B_{t}\right)}{\left(X-\xi^{t}\right.} d \xi\right\}^{* *} \\
& =-(i k)^{\frac{1}{2}\left(k^{\prime}+l^{\prime}\right)^{\frac{1}{2}}\left(A_{0}+B^{* *} .\right.}
\end{aligned}
$$

A (rully-implicit) Crank-Nicolson scheme was applicd to (3.7) with the initial conditions (at $t=0$ ) prescribed by the corresponding steady solution for $\gamma(Z, t=0)$, using the computer code developed for $I$.

He restricted attention to forcing functions of the form

$$
\gamma(Z, t)=\gamma_{1}(Z) \gamma_{2}(t),
$$

which enabled the various "invariant" functions (e.g. Ao) to be evaluated just once, prior to the unsteady computation commencing.

One additional feature here, not present in $I$, is the inclusion of the time derivative term. At each timestep (at the ith $X$ location) we make the following approximation 


$$
\begin{aligned}
& \int_{-\infty}^{X_{i}} \frac{B_{t}(\xi, Z, t-\Delta t / 2) d \xi}{\left(X_{i}-\xi\right)^{t}} \\
& \simeq \int_{-\infty}^{X_{i}-\Delta X} \frac{B_{t}\left(\xi, Z, t-\frac{\Delta t}{2}\right)}{\left(X_{i}-\xi\right)^{t}} d \xi \\
& +(\Delta X)^{3 / 4}\left\{\frac{2}{33} B_{t}\left(X_{i}, Z, t-\frac{\Delta t}{2}\right)\right. \\
& -\frac{8}{77} B_{t}\left(X_{i}+\Delta X, Z, t-\frac{\Delta t}{2}\right) \\
& \left.+\frac{36}{77} B_{t}\left(X_{i}-\Delta X, Z, t-\frac{\Delta t}{2}\right)\right\} .
\end{aligned}
$$

where $\Delta X$ is the grid size in the $X$ direction (and $\Delta Z$ the grid size in the $Z$ direction), and $\Delta t$ the time step. The Crank-Nicolson diffencing scheme made the following approximation

$$
\begin{aligned}
B_{t}\left(X_{i}, Z, t-\frac{\Delta t}{2}\right) \\
\simeq=\frac{B\left(X_{i}, Z, t\right)-B\left(X_{i}, Z, t-\Delta t\right)}{\Delta t},
\end{aligned}
$$

whilst the integral term in (3.19) was evaluated using a standard trapezoidal scheme (coupled with (3.10)).

The term involving $B$ on the right-hand-side of (3.7) was evaluated using the fast Fourier Transform tcchnique of Cooley and Tukcy (1965), in which the function is actually evaluated in $\operatorname{spectral}(k, l)$ space, and then transformed back into physical $(X, Z)$ space; this same lcchnique was employed in I, and results in considerable savings on computational times.

At each time and itcration level, at each $Z$ location, the (algebraic) approximation to (3.7) can be written symbolically as

$$
\underset{\sim}{\mathrm{B}^{2}}+\underset{\sim}{\mathrm{B}}=\underline{\mathrm{R}} \text {. }
$$

where $I$ is the unit matrix and $\underset{B}{B}=B\left(X_{i}(i=1, N), Z, l\right)$. We then write 


$$
\underline{B}=\underline{B}^{(n-1)}+\delta \underline{B}
$$

where $\underset{\sim}{B}(n)$ denotes the value at the $n^{\text {th }}$ iteration level; here we discard $0\left(\mid \delta \mathrm{\sim}_{\mid}{ }^{2}\right)$ terms, to yield the system

$$
\begin{aligned}
(2 \underline{\sim} & \left.\underline{\sim}^{(n)}+\underline{E}\right) \delta B \\
& =\underset{\sim}{R}-\underset{\sim}{\mathrm{B}}(n)^{2}-\underline{E} \underline{\sim}^{(n)}
\end{aligned}
$$

Thus to obtain a new estimate for the $\underline{B}^{(n)}$ a linear algebraic system was solved (in I, just a scalar system was solved; the off-diagonal terms in (3.13) arise directly from the time derivative term.) This scheme is loosely based on Newton's method. The solution was then determined at all $Z$ stations, and the overall proccdure then repcated until convergence was attained, where-upon the calculation proceeded to the following timestep. 


\section{Numerical Solutions}

In this section we present numerical solutions for two choices of $\gamma(Z, t)$ (and, as it turns out these exhibit quite different features). The first example taken was with $\lambda=S=1$ and

$$
\gamma(Z, t)=e^{-Z^{2}}\left(-2+4 \tanh ^{2} t\right) \text {, for } t \geq 0 \text {, }
$$

and hence we have

$$
\begin{aligned}
& \gamma_{1}(Z)=\mathrm{e}^{-Z^{2}}, \\
& \gamma_{2}(\mathrm{t})=-2+4 \tanh ^{2} \mathrm{t}, \mathrm{t} \geq 0 .
\end{aligned}
$$

In this example (and in the following example) conditions at $1=0$ were obtained by solving the sleady problem (considered in I) with $\gamma(\mathrm{Z}, 0)$. Further, since $\gamma_{2}(\mathrm{t})$ varics (monotonically) from -2 at $t=0$ to +2 as $t \rightarrow \infty$, then according to $I$, the corresponding steady problem with (4.2) lies entirely within the envelope of steady solutions found in $I$.

Figure 1 shows the temporal variation of $A(X, Z, t)$ at selected $X$ and $Z$ stations, and these confirm an evolution from the initial steady state $\gamma_{1}(t=0)=-2$ to the final $\gamma_{1}(t>1)=+2$ state (and Fig. 1 shows results agreeing with the corresponding steady "upper branch" solution in this limit). Interestingly, a common trend observed at the locations considered in Fig. 1 is that, although $\gamma(Z, t)$ reduces monotonically with time (as would quasi-steady results for $A(X, Z)$, based on the results of I), there is scen to be a very slight "tough" in the results, soon after the computation commences, followed by a very pronounced peak soon after $t=1$, followed by a monotonic decrease towards the $t \gg 1$ values. This particular computation was carried out using a grid which extended from $X$ (and $Z$ ) $\simeq-10.32$ to $X$ (and $Z$ ) $\simeq 9.68$ with $\Delta X=\Delta Z \simeq 0.64, \Delta t=0.005$ and $\alpha_{0}=1$; we shall refer to this as grid I.

The second (and what turns out to be the more challenging and 
interesting) example taken was $\lambda=S=1$ with

$$
\gamma(z, t)=e^{-Z^{2}}\left(-2+8 \tanh ^{2} t\right), t \geq 0
$$

and hence $\gamma_{1}(Z)$ is given by (4.2), whilst

$$
\gamma_{2}(t)=-2+8 \tanh ^{2} t, t \geq 0 \text {. }
$$

Thus we see $\gamma_{2}(t)$ extends from -2 at $t=0$ (as in the previous example) to +6 as $t+\infty$. However this latter value lies outside of the envelope of stcady solutions found in I corresponding to our choice of $\gamma_{1}(Z)$.

The distribution of $A(X, Z)$ at fixed $X$ or $Z$ stations and fixed t are shown in Figs.2 - 5. These particular computations were performed on a grid that extended from $X$ (and $Z$ ) $\simeq-10.15$ to $X$ (and $Z$ ) $\simeq 9.84$, with $\Delta X=\Delta Z \simeq 0.32, \Delta t=0.005$ and $\alpha_{0}=1$; we refer to this as grid II .

It is quite clear that a singularity is forming, close to $X=Z=0$, at a finite time, with $A(X, Z, t)$ becoming progressively larger and more negative at this location. Figure 6 shows the temporal variation of $A(X=Z=0)$ on a variety of grids, to enable a (partial) assessment to be made of the accuracy of the scheme in this critical region. The solidus denotes the grid II results. The broken line - - represents the grid I results (a computation was also carried out on this grid, but with $\alpha_{0}=3$; these proved to be indistiguishable on the scale used in Fig. 6). The dot-dashed line - . - denotes grid III results with $X$ and $\mathrm{Z}$ extending from (approximatly) -5.16 to +4.84 with $\Delta X=\Delta Z \simeq 0.32, \Delta t=0.005$ and $\alpha_{0}=1$. Grid IV results are shown as a dotted line ....... a grid which is identical to grid I. except $\Delta t=0.05$. All these results confirm the likely appearance of a finite-time singularity close to $x=Z=0$; the accuracy of the computations is also confirmed.

Figure 7 shows the (spectral) distribution of $\operatorname{Re}\left\{B^{* *}(k, l=0, l)\right\}$ 
with $k$ at selected times, (computed on grid Il) It appears that this distribution (and indeed other spectral distributions, not shown here) undergoes a rapid change in behaviour as the apparent breakdown is approached. In particular the decay of the solution as $|k| \gg l$ is seen to diminish (and this in turn mirrors the finite-time, finite-location breakdown in physical $X, Z$ space).

In the following section we go on to analyse the structure of this likely singularity. 


\section{The breakdown}

We now seek to determine the nature of the breakdown suggested in a number of the numerical results described in the previous section.

In particular, we seek an asymptotic solution which predicts $|A| \rightarrow \infty$ at a finite value of $X\left(X_{0}\right.$ say), at a finile value of $Z$ $\left(Z_{0}\right.$ say): (notice that the indications from the results for the configuration in the previous section that resulted in a breakdown are that $z_{0} \simeq 0$ ), at a finite line ( $t_{s}$ say). We define the (small) t imescale

$$
\tau=t_{s}-t
$$

then inspection of (3.7) suggests that if the (i) non-linear, (ii) time derivative and (i i i) the right-hand-side terms all balance, then the following ("hatted") variables are the most appropriate

$$
\begin{aligned}
& x-X_{0}=\tau^{4 / 9} \mathrm{~s}^{-4 / 9} \hat{X} \\
& z-z_{0}=\tau^{4 / 9} \mathrm{~s}^{-4 / 9} \hat{\mathrm{Z}} \\
& \mathrm{A}=\tau^{-2 / 3} \mathrm{~s}^{2 / 3} \hat{\mathrm{A}}(\hat{\mathrm{X}}, \hat{\mathrm{Z}})+o(\tau-2 / 3) .
\end{aligned}
$$

Substitution of these forms into (3.3), taking the largest terms in $\tau$, and writing the solution entirely in physical $(\hat{X}, \hat{Z})$ space yields the following system

$$
\begin{aligned}
& \hat{A}^{2}+\frac{4}{9 \Gamma(3)} \int_{-\infty}^{\hat{X}} \frac{\frac{3}{A}+\hat{\xi} A \hat{\xi}+\hat{\zeta} A \hat{\zeta}}{(\hat{X}-\hat{\xi})^{t}} d \hat{\xi} \\
& =\frac{8}{\pi} \int_{-\infty}^{\infty} \int_{-\infty}^{\infty} \frac{[\hat{A} \hat{\xi} \hat{\xi} \hat{\xi}+\hat{A} \hat{\zeta} \hat{\zeta} \hat{\zeta}]}{\left[(\hat{X}-\hat{\xi})^{2}+(\hat{Z}-\hat{\zeta})^{2}\right]} K(\chi) d \hat{\xi} d \hat{\zeta} .
\end{aligned}
$$

where

$$
\chi=\left\{1+\frac{\hat{X}-\hat{\xi}}{\left[(\hat{X}-\hat{\xi})^{2}+(\hat{Z}-\hat{\zeta})^{2}\right]^{t}}\right\}^{\frac{1}{2}},
$$


and $K(\chi)$ denotes the complete elliptic integral of the first kind, argument $\chi$.

In order to match correctly on to the $X=0(1), Z=0(1)$ problem, we require that

$$
A \sim a(\hat{X} / \hat{Z})\left(\lambda_{1} \hat{X}^{2}+\hat{Z}^{2}\right)-3 / 4
$$

as $\hat{X}^{2}+\hat{Z}^{2} \rightarrow \infty$, where $\lambda_{1}$ is a constant and $a(\hat{X} / \hat{Z})$ is a function of $\hat{X} / \hat{Z}$. Both quantities must be determined from the terminal bchaviour of (3.2). (The scalings (5.2) are entirely consistent with the twodimensional work of Smith 1982).

Indeed, it is possible to obtain a result identical to (5.5) using a linearized form of (3.2). This may be justified by supposing that the parameter $S \gg 1$, i.e. we are concerned with rapid temporal variations in external forcing. We also suppose that the typical $X$ and $Z$ lengthscales are both $0\left(S^{-4 / 9}\right)$. The resulting system is then (having scaled out $S$ )

$$
A_{t}^{* *}+\left(k^{2}+l^{2}\right) \frac{1}{t}(i k)^{5 / 4} A^{* *}=0 \text {. }
$$

The solution to this is

$$
A^{* *}=A_{0}^{* *}(k, l) e^{\left(k^{2}+l^{2}\right)^{\frac{1}{2}}(i k)^{5 / 4}\left(t-t_{s}\right)} \text {. }
$$

From this we see that as $t \rightarrow t_{s}$, then the solution approaches a finitetime breakdown, with the appropriate $X$ and $z$ scales being $0\left(\left(t_{s}-t\right)^{9}\right)$, in both cases, in complete accord with (5.2).

Let us now return to consider the systcm (5.3); this, in general, requires a fully numerical (and quite substantial) task. However, we can make some further analytic progress (that suggests the existence of regular solutions to (5.3) for all $\hat{X}$ and $\hat{Z}$ ), by using the idea, put forward by Smith (1982), of assuming $|\hat{A}| \ll 1$ for all $\hat{X}$ and $\hat{Z}$ (this amounts to assuming $|a(\hat{X} / \hat{Z})| \ll 1)$. With this restriction the $\cdot \hat{A} 2$. lerm on the left-hand-side of (5.3) may be discarded. If the resulting 
system is subject to a double Fourier transform, then (after

normalising 10 remove positive constants, and then using tilde variables)

$$
\begin{aligned}
\pm \tilde{A}^{* *} & +\tilde{\mathbf{k}} \frac{\partial \tilde{A}^{* *}}{\partial \tilde{\mathrm{k}}}+\tilde{\ell} \frac{\partial \tilde{A}^{* *}}{\partial l} \\
& =(i \tilde{\mathrm{k}})^{5 / 4}\left(\tilde{\mathrm{k}}^{2}+\tilde{l}^{2}\right)^{\frac{1}{2}} \tilde{A}^{* *} .
\end{aligned}
$$

This may be solved using a technique based on the method of characteristics; defining variables $\xi_{1}=\left(\tilde{\mathrm{k}}^{2}+\lambda_{1}^{2} \tilde{\mathrm{l}}^{2}\right)^{t}, \quad \xi_{2}=\overline{\mathrm{k}} / \overline{\mathrm{l}}$; $(5.8)$ may then be written in the form

$$
\xi_{1} \frac{\partial \tilde{A}^{* *}}{\partial \xi_{1}}=\left\{-\frac{1}{2}+\frac{\left(i \xi_{1}\right)^{5 / 4 \xi_{1}}}{\left(1+\frac{\lambda_{1}{ }^{2}}{\xi_{2}^{2}}\right)^{5 / 8}}\left[\frac{\xi_{2}{ }^{2}+1}{\xi_{2}{ }^{2}+\lambda_{1}{ }^{2}}\right]^{\frac{1}{2}}\right\} \tilde{A}^{* *} .
$$

This can be integrated routincly to give the following general solution $\tilde{A}^{* *}(\tilde{k}, \tilde{l})=F^{* *}\left(\frac{\tilde{k}}{\tilde{l}}\right)\left(\tilde{k}^{2}+\tilde{\lambda}_{1}^{2} \tilde{l}^{2}\right)^{-1} \exp \left\{-\frac{4}{9}(i \tilde{k})^{5 / 4}\left(\tilde{k}^{2}+\tilde{l}^{2}\right)^{\frac{1}{2}}\right\}$.

Here $F^{* *}(\tilde{k} / \tilde{l})$ is an arbitrary function of $(\tilde{k} / \tilde{l})$. The solution $(5.10)$ is cntirely consistant with (5.5) (seen by allowing $k^{2}+\ell^{2} \rightarrow 0$ ). The importance of $(5.10)$ is that it illustrates that a regular solution exists to (the linearised form of) (5.3) for all $\hat{x}$ and $\hat{z}$, and as such strongly suggests that the terminal description of the breakdown is given by the solution of (5.3). 


\section{General Discussion}

In this paper (i) we have presented the structure of the flow in the vicinity of a threc-dimensional marginal separation point (situated along a line of symmetry), including the effects of unsteadiness at this point; ( $i$ i) the solution of the non-linear, unsteady integrodifferential system has been carried out in two distinct cases, the first where the flow evolves regularly from one steady state to another steady state: and the second where a three-dimensional breakdown is scen to be observed; (ii i) the structure of this breakdown has also been described.

Although, from the point of view of this paper, the particular details of the mechanism by which unsteadiness is injected into the problem are not important, we could, perhaps envisage this may be achieved (for example) by a pitching/buffeting of an aerofoil/ or a ae rodynamic body.

Qualitative comparison between the numerical results of Section 4 , with the analysis of Section 5 secms quite supportive, although the computations do become very much more difficult as the breakdown is approached (see the remarks of Smith 1982). Certainly the numerical results point to the emergence of a small region (close to $X=Z=0$ ) inside of which $A(X, Z, t)$ increases rapidly, both spatially and temporally.

The implications of this breakdown are that as $\gamma(Z, t)$ increases above its critical value although the flow initially responds slowly (on a timescale $0\left(\frac{1}{20}\right)$ ), the flow then develops suddenly/explosively as the breakdown time is approached.

A further matter concerns the ulimate behaviour of the flow. leading from the breakdown detailed in Section 5 . It is surmised by Smith (1982) that (in the two-dimensional context) the breakdown leads ultimately to the flow being governed by a form of the unsteady boundary-layer equations. Here, in the three-dimensional situation, such 
a scenario seems not unreasonable. This aspect is currently under invest igation. 


\section{Acknowlegements}

Much of this work has carried out whilst the author was visiting NASA Lewis Research Center (under the ICOMP Program). A number of computations were carried out at Manchester Computer Centre with Computer t ime provided under SERC Grant no, GR/E/25702. 


\section{References}

Brotherton-Radcliffe, R and Smith, F.T. 1987. Complete breakdown of an unsteady interactive boundary layer (on a surface distribution or in a liquid layer) Mathematika 34,86 .

Brown, S.N. 1985. Marginal separation of a three-dimensional boundary layer on a line of symmetry. J. Fluid Mech. 158, 45.

Brown, S. N. and Stewartson. K. 1983. On an integral equation of marginal separation. SIAM J. Appl. Maths. 43, 1119.

Cebeci, T., Khat tab, A.K. and Stewartson, K. 1980. On nose separation J. Fluid, Mech. $97,435$.

Cebeci, T, and Su, W. 1988. Separation of three-dimensional laminar boundary layers on a prolate spheroid J. Fluid Mech. 191, 47.

Cooley, J.W. and Tukey, J.H. 1965. An algorithm for the machine computation of complex Fourier Series, Maths. Comp. 19, 297.

Duck, P.W. 1985a. Pulsatile flow in constricted or dilated channels. Part II. Quart. J. Mech. App. Math. 38, 621.

Duck P.W. 1985b. Laminar flow over unsteady humps; the formation of waves. J. Fluid Mech. 166, 465.

Duck. P.W. 1987. Unsteady triple-deck flows leading to instabilities. Proc. IUTAM. Symp. on Boundary-layer Separation 1986, 297, Springer. 
Duck, P.W. 1989. Three-diemsional marginal separation. J. Fluid Mech. 202, 559 .

Duck, P.W. 1990. Triple-deck flow over unsteady surface distributions: the three-dimesional development of Tollmien Schlichting waves. Computers and Fluids, 18, 1 .

Duck, P.W. and Burggral O.R. 1986. Spectral solutions for threedimensional triple-deck flow over surface topography. J. Fluid Mech. 162, 1.

Elliott, J.W. and Smith, F. T. 1987. Dynamic stall due to unsteady marginal separation. J. Fluid. Mech. 179, 489.

Goldstein, M.E., Leib, S.J. and Cowley, S.J. 1987. Generation of Tollmien-Schlichting waves on interactive marginally separated flows. J. Fluid. Mech. 181, 485 .

Ryzhov, 0.S. and Smith, F.T. 1984. Short-length stabilities, breakdown and initial value problems in dynamic stall. Hathematika $31,163$.

Smith, F.T. 1982. Concerning dynamic stall. Aero. Q. 33, 331.

Smith, F.T. 1988. Finite-time breakdown can occur in any unsteady interacting boundary layer. Mathematika 35, 256 .

Stnith, F.T. and Bodonyi, R.J. 1985. On short-scalc inviscid instabilities in flow past surface - mounted obstacles and other non-parallel motions Acro. J. 89, 205. 
Smith, F.T. and Elliott, J.W. 1985. On the abrupt turbulent reattachment downstream of a leading edge separation. Proc. R. Soc. Lond. A 394, 25 .

Smith, F.T., Sykes, R.I. and Brighton, P.W.M. 1977. A two-dimensional boundary layer encountering a threc-dimensional obstacle. J. Fluid Mech. 83, 163 .

Stewartson, K. 1970. Is the singularity at separation removable? J. Fluid Mech. $44,347$.

Stewartson, K., Smith, F.T. and Kaups, K. 1982. Marginal separation Stud. Appl. Maths. 67, 45 .

Tutty, O.R. and Cowley, S.J. 1986. On the stability and numerical solution of the unsteady boundary-layer equation. J. Fluid. Mech. 168, 431 .

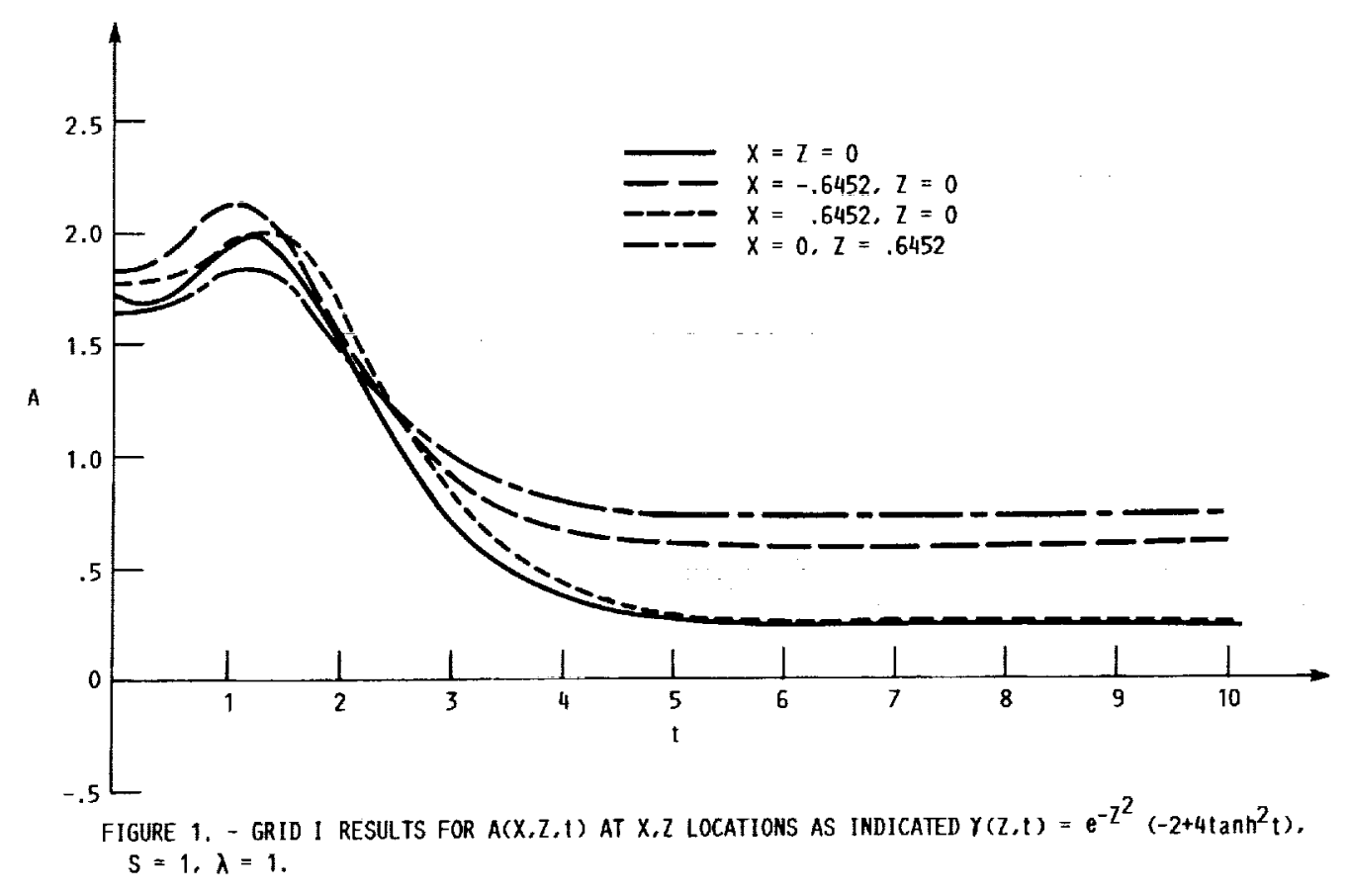




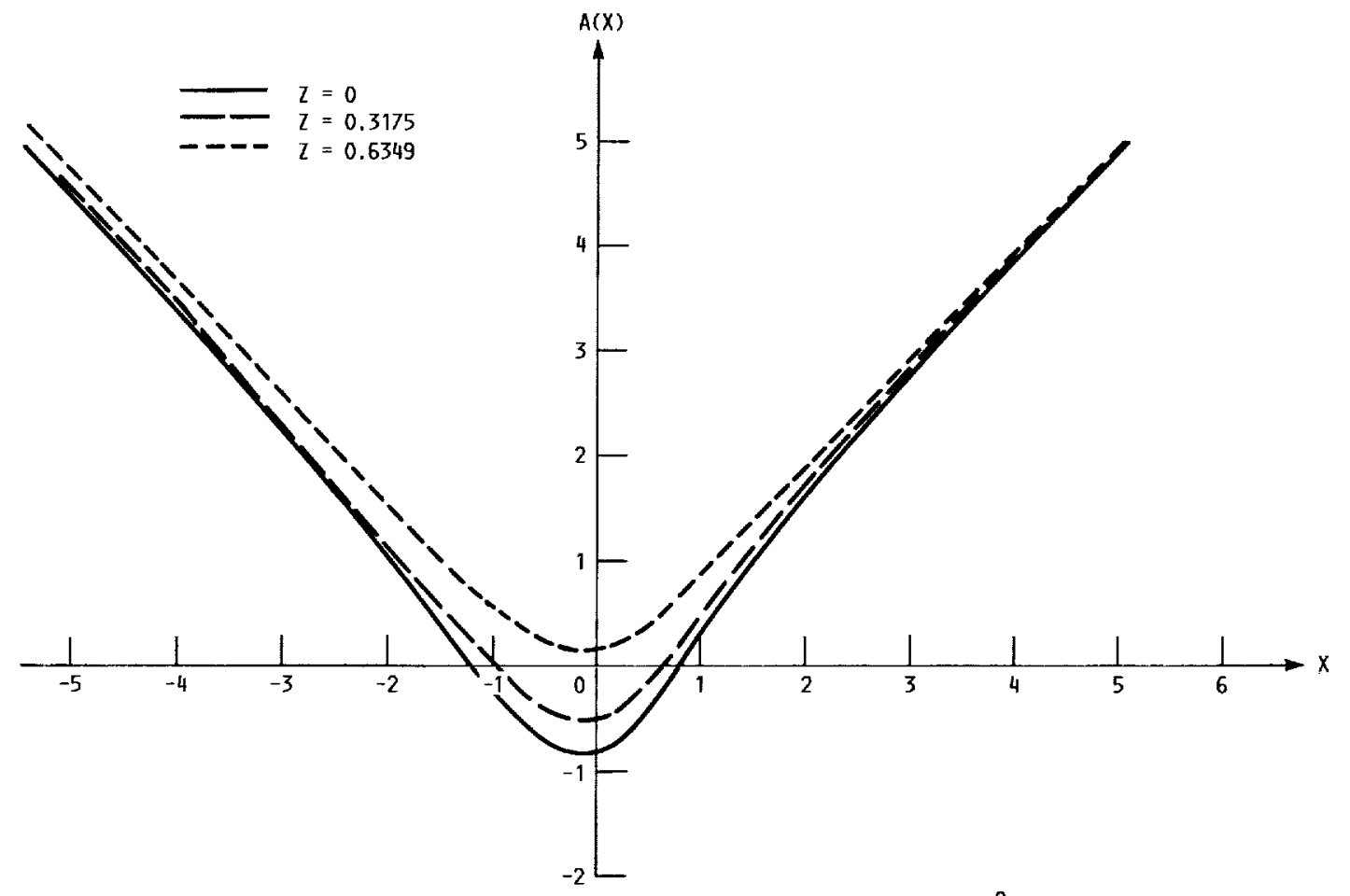

FIGURE 2. - SPATIAL VARIaTION OF $A(X, Z, t=3), s=1, \lambda=1, \gamma(Z, t)=\mathrm{e}^{-Z^{2}}\left(-2+8 \tanh ^{2} \mathrm{u}\right)$.

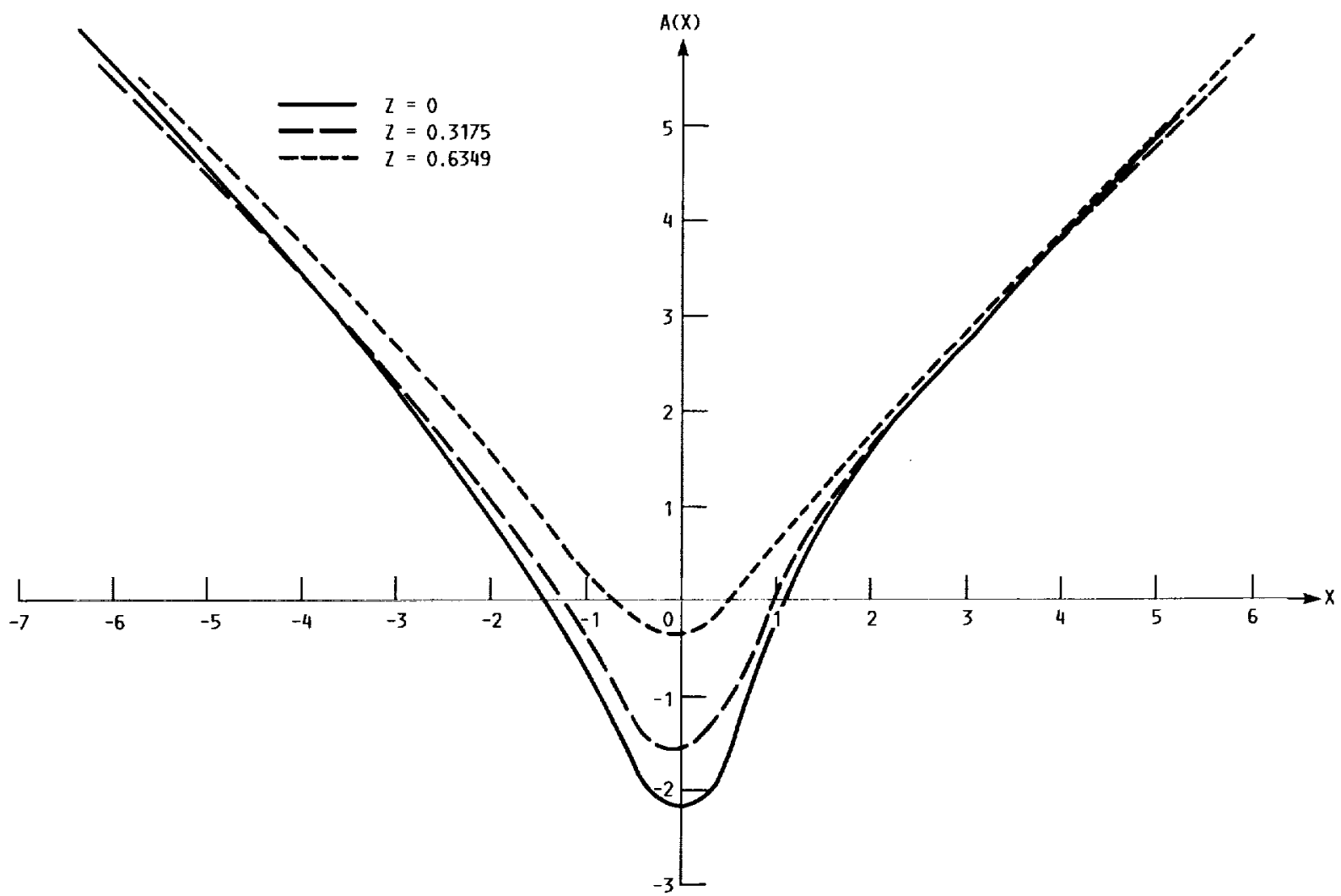

FIGURE 3, - SPATIAL VARIATION OF $A(X, Z, t=3,365), s=1, \lambda=1, \gamma(Z, t)=e^{-Z^{2}}\left(-2+8 \tanh ^{2} t\right)$. 


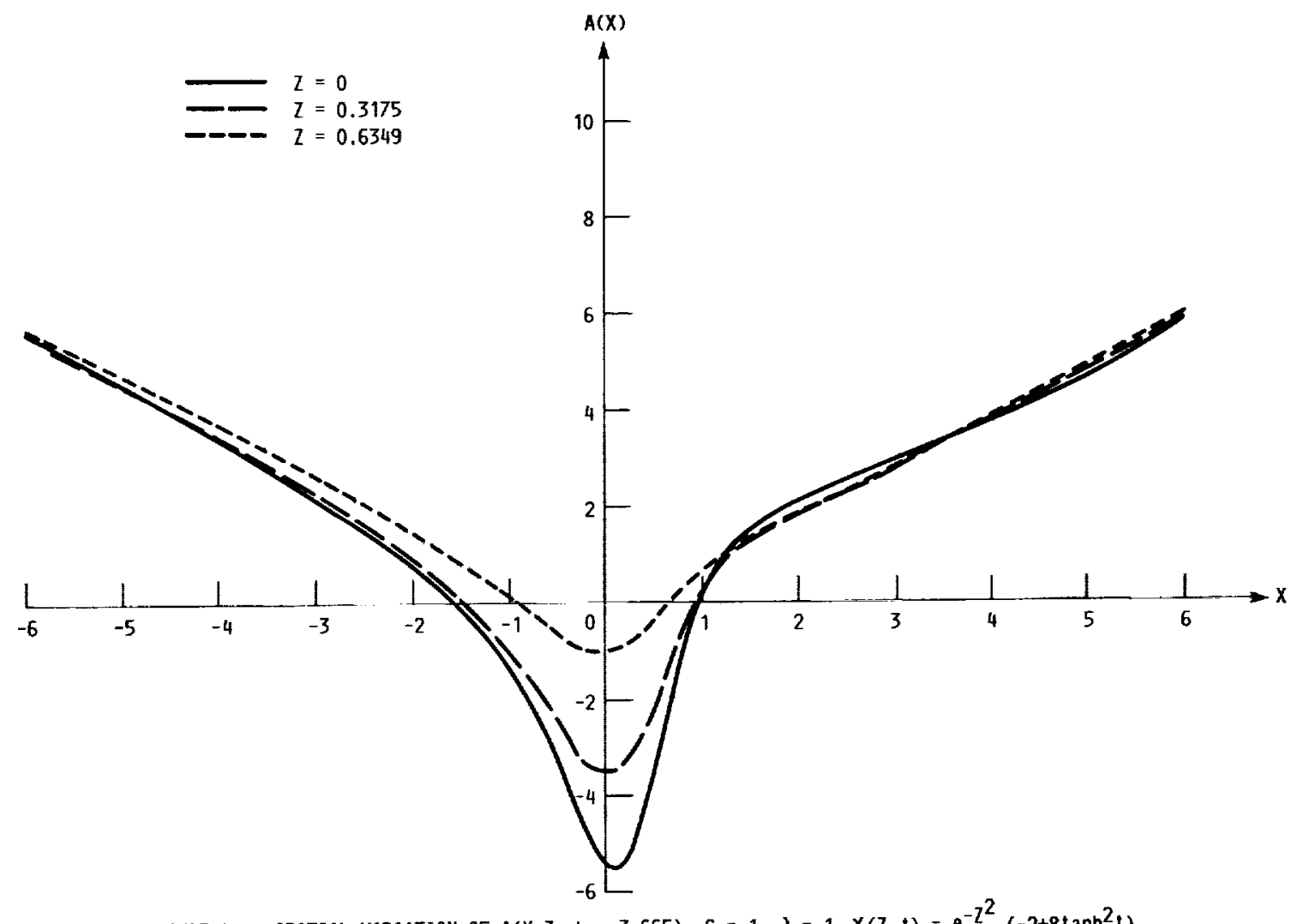

Figure 4. - Spatial variation of $A(x, z, t=3.665), s=1, \lambda=1, y(z, t)=e^{-z^{2}}\left(-2+8 \tanh ^{2} t\right)$.

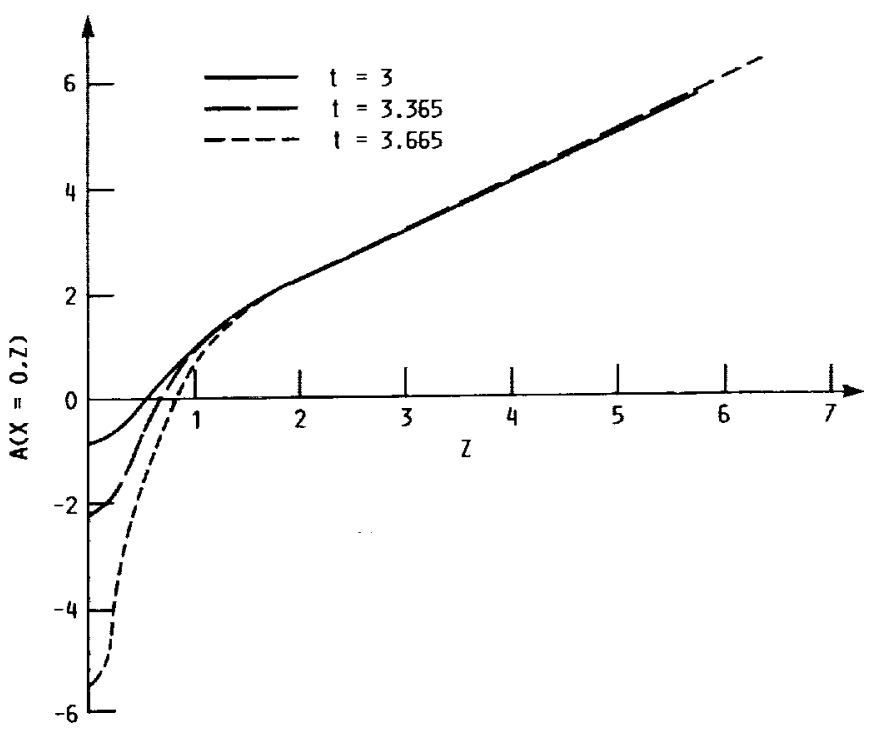

FIGURE 5. - SPATIAL VARIATION OF $A(X=0,2, t), S=1, \lambda=1$, $Y(z, t)=\mathrm{e}^{-z^{2}}\left(-2+8 \tanh ^{2} t\right)$. 


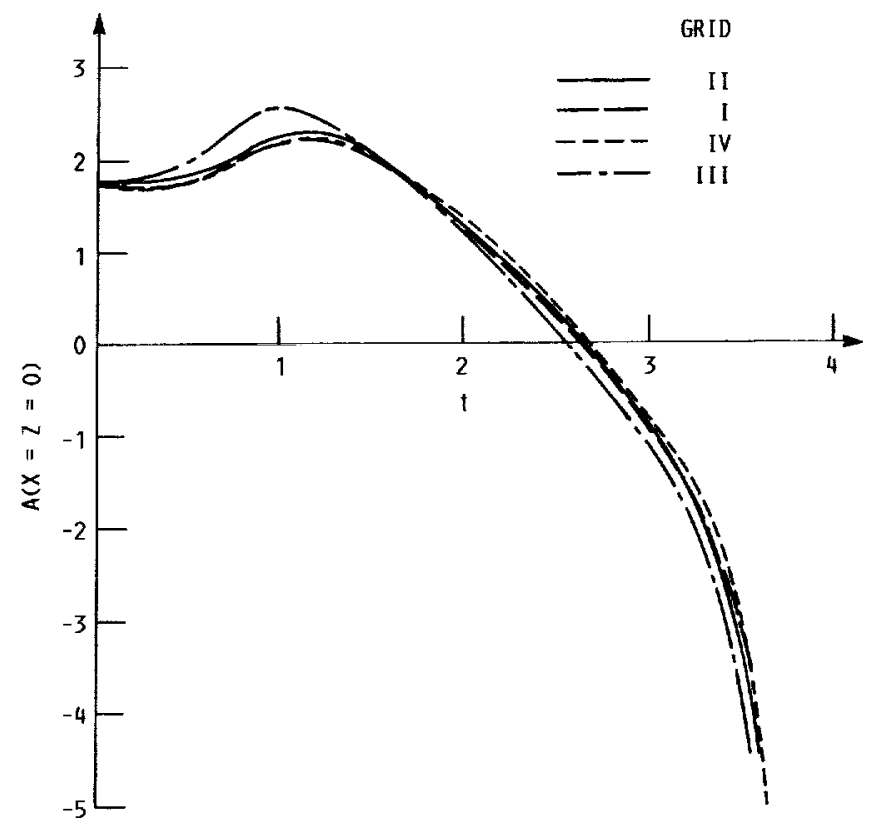

FIGURE 6. - TEMPORAL VARIATION OF $A(X=Z=0,1), S=1$. $\lambda=1, \gamma(z, t)=e^{-z^{2}}\left(-2+8 \tanh ^{2} t\right)$.

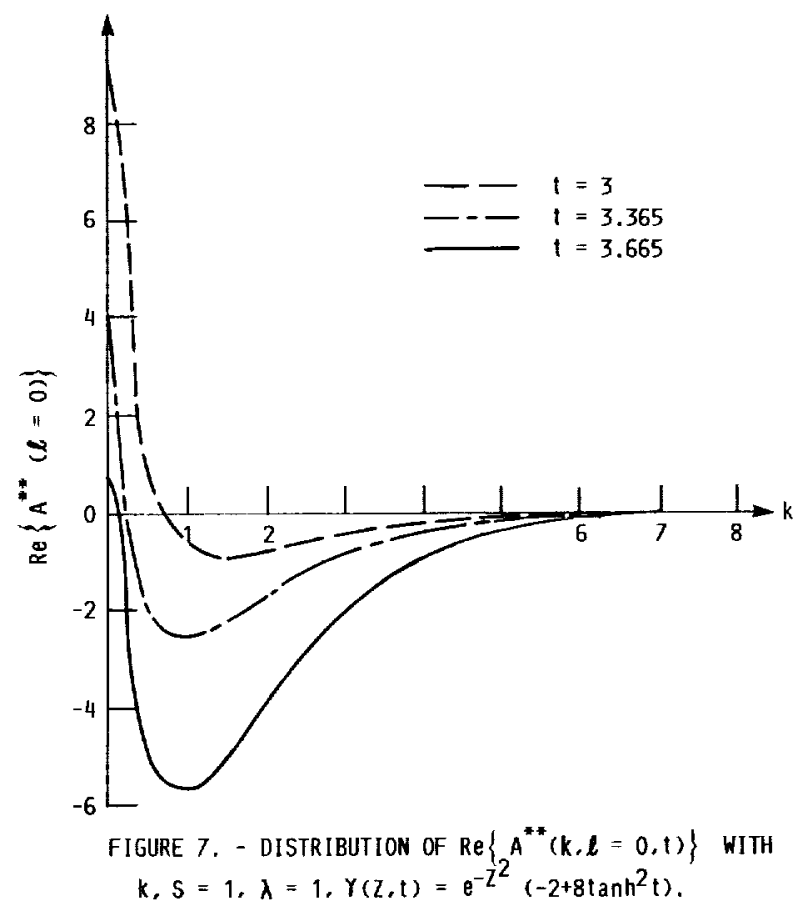




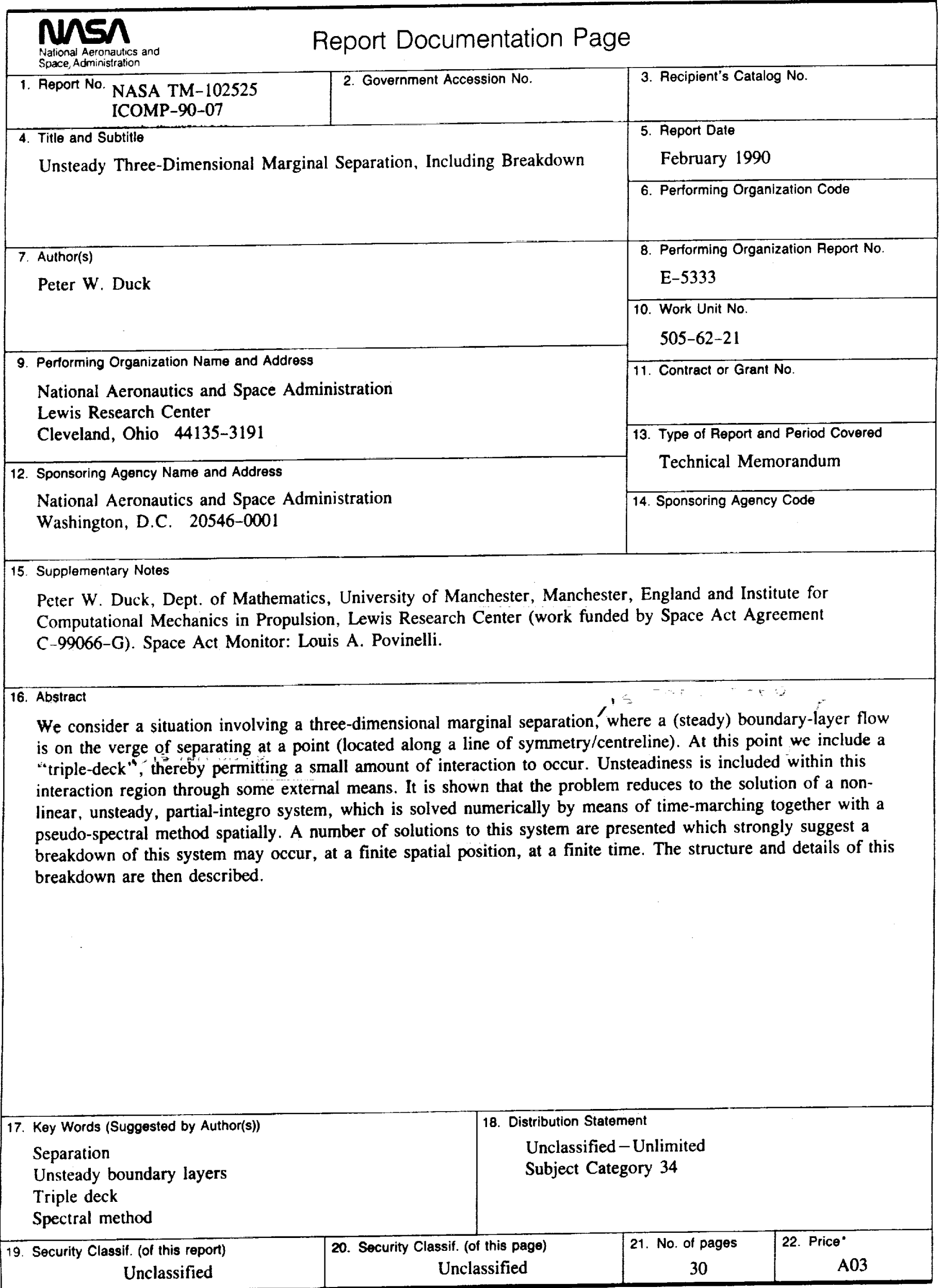

